\title{
MRAP2 in the Hypothalamus of Prenatally Betamethasone Primed Rats and Saline Controls for Treatment of Infantile Spasms
}

\author{
Nitin Seshadri \\ Somers High School, 120 Primrose St., Lincolndale, NY, 10505, U.S.A.; seshadri.nitin@somersschools.org
}

\begin{abstract}
Infantile Spasms (IS) are an epilepsy syndrome of infancy, present in 1 out of every 3,000 live births. They typically affect infants younger than 2, with a $15-30 \%$ mortality rate, and up to $85 \%$ of survivors suffer from cognitive impairments. The hypothalamic-pituitary-adrenal (HPA) axis is impaired in IS patients, causing decreased adrenocorticotropic hormone (ACTH) levels. ACTH intramuscular injections are currently used as a first-line treatment to mitigate this, but they are not effective in $45 \%$ of patients. Melanocortin receptor accessory protein 2 (MRAP2) assists ACTH in binding to melanocortin (MC) receptors and suppressing spasms. For this $45 \%$, boosting MRAP2 expression would allow ACTH to bind better, improving the ACTH response. We hypothesize that prenatal betamethasone priming enhances MRAP2 expression. We measured MRAP2 in the arcuate (ARC) and paraventricular (PVN) nuclei of the hypothalamus. Results were compared between rats prenatally primed with betamethasone and controls exposed to saline, and there was a statistically significant difference in cell area density in ARC in prenatally betamethasone primed rats. We may be able to boost the activity of MRAP2 by specific agonists or the expression by molecular biology tools to improve the ACTH response and help the $45 \%$ of patients be spasm-free.

KEYWORDS: Infantile Spasms; Hypothalamus; ACTH; MC2R; MRAP2; Betamethasone.
\end{abstract}

\section{- Introduction}

Infantile spasms (IS), also known as West Syndrome, are catastrophic epileptic seizures that usually develop between 3 months to 1 year of age. ${ }^{1}$ One out of every 3,000 live births will result in a diagnosis of infantile spasms. ${ }^{2}$ The mortality rate is $15-30 \%$, with up to $85 \%$ of surviving patients affected by mental deterioration. Visible symptoms include uncontrollable flexion and extension of arms and legs. The spasms frequently occur upon awakening and can occur hundreds of times each day. The syndrome is characterized by a specific brain pattern produced on an electroencephalogram (EEG) a device used to track and record brain wave patterns called hypsarrhythmia: chaotic, asynchronous, high amplitude waves recorded from the entire skull surface. The spasms usually disappear by age 5 but may lead to other seizure types such as the more severe Lennox-Gastaut syndrome and increased risk for autism. ${ }^{3}$

In a healthy infant, the hypothalamus stimulates the pituitary gland with a corticotropin-releasing hormone to release ACTH or adrenocorticotropic hormone, a peptide hormone. It maintains homeostasis during periods of stress by sending messages through the melanocortin $(\mathrm{MC})$ receptor proteins and these messages in turn act on the adrenal glands.

However, in IS patients, this hypothalamic-pituitary-adrenal (HPA) axis is impaired, causing decreased ACTH levels, 4 so $\mathrm{ACTH}$ intramuscular injections are currently used as a first-line of treatment. ${ }^{1}$

Though this treatment is widely used, it is not effective in $45 \%$ of patients. In these patients, ACTH does not bind effec- tively to its receptors and therefore does not suppress spasms. ${ }^{5}$ It is in this context that a need for a better understanding of the pathways of ACTH arises.

Our research indicates that prenatal betamethasone priming enhances expression of the melanocortin receptor accessory protein MRAP2, which allows ACTH to reach its receptors and actively suppress infantile spasms. MRAP2 expression is significantly enhanced in the ARC region of the hypothalamus but not enhanced in the PVN region of the prenatally betamethasone primed rats in vivo.

\section{Review of Literature}

Peptide hormones are carried into and out of the cells by protein receptors or receivers. By manipulating such receptors, it is possible to determine their role and impact so that hormones can be increased or inhibited to actively change the response of the body. ${ }^{6}$ In addition, there are other proteins, called accessory proteins, that either act as an agonist (enabler) or an antagonist (inhibitor) for the receptors, and aid or inhibit the function of the receptor. ${ }^{6}$

G-protein coupled receptors (GPCRs) are regulated by numerous proteins including kinases, $G$-proteins, $\beta$-arrestins, and accessory proteins. Several families of GPCR accessory proteins such as Receptor Activity Modifying Proteins, Receptor Transporting Proteins, and Melanocortin Receptor Accessory 
Proteins (MRAPs) have been identified as regulators of receptor trafficking, signaling and ligand specificity. ${ }^{6}$

The MRAP family contains two members, MRAP1 and MRAP2, which are responsible for the formation of a functional melanocortin receptors (MCRs) and the regulation of energy homeostasis, respectively. Like all known GPCR accessory proteins, MRAPs are single transmembrane proteins, however, they form a unique structure since they assemble as an antiparallel homodimer. ${ }^{7}$ The earlier understanding that MRAPs only regulate melanocortin receptors was challenged by the discovery that MRAP2 also inhibits the activity of prokineticin receptors. Recent studies are starting to explain the role of the unusual structure of MRAPs and to illustrate the importance of MRAP2 for the maintenance of energy and glucose homeostasis. ${ }^{6}$ See Figure 1 for an illustration of the MRAP2 protein and how it enables MC1R through MC5R receptors. Figure 2 shows the anti-parallel homodimer structure of MRAP2 proteins.

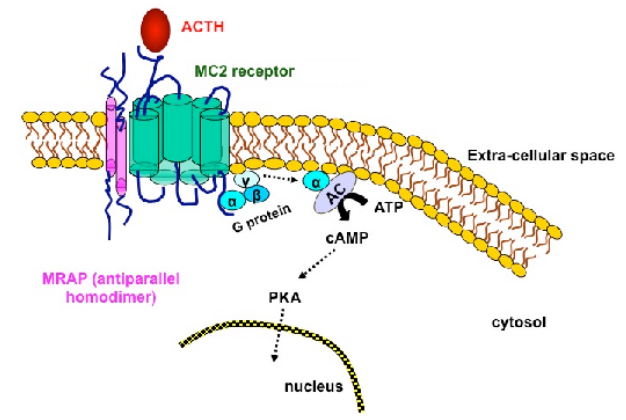

Figure 1. MRAP2 as a regulator of an ACTH Receptor. ${ }^{11}$

MRAP1 and MRAP2 are accessory proteins that are found to interact and control receptors primarily the melanocortin 2 receptor (MC2R). The MC2R receptors are occupied by the ACTH that is released from the pituitary gland of the brain. $\mathrm{ACTH}$ and the MC2R are primarily responsible for the interactions and signaling tied to the synthesis and release of corticosteroids, energy use, body length, and glucose metabolism, all of which are directly related to body growth and obesity as seen in multiple studies. ${ }^{8}$

Between the 2 MRAPs, MRAP2 seems to be more complex as it not only interacts with MC2R but also with other G-proteins, such as the Kir7.1 protein. MRAPs are sometimes dubbed "promiscuous" as a result. ${ }^{6}$ Research done by other scientists has also shown that MRAP2 interactions are much more complex and there are multiple pathways through which MRAP2 is observed to work and hence reinforced the term "promiscuity" of the MRAPs. Taken together, both MRAP1 and MRAP2 are implicated with glucose and energy balance and regulation within the body.

Melanocortin 2 Receptor Accessory Protein (MRAP) was originally discovered as an essential co-receptor for ACTH/ Melanocortin 2 receptors. A related molecule called MRAP2 is mainly expressed in the central nervous system (CNS) and

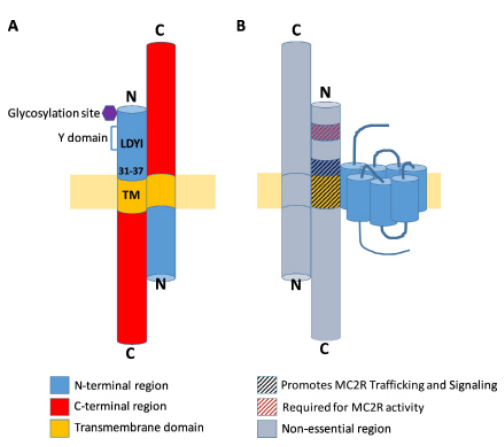

Figure 2. Schematic representation of MRAP1 structure and domains. A. Representation of MRAP1 antiparallel homodimer. B. Localization and function of important MRAP1 domains for MC2R trafficking and/or signaling. ${ }^{6}$

seems to have a role as an accessory protein to the Melanocortin 4 receptor. Though Mrap2 knockout mice (where the Mrap2 gene is removed) have shown to become obese, other characteristics of this phenotype differ from MC4R deleted mice. ${ }^{9}$ So, the MRAP and MRAP2 accessory proteins may have a wider role than in melanocortin receptors alone. ${ }^{10}$

MRAPs are also shown to interact with melanocortin protein receptors as well as the transportation of prokineticin receptor 1. However, MRAP2 is shown to be more complex and multi-faceted than in its workings in prior research. MRAP2 is also shown to regulate another receptor called Orexin Receptor 1 (OX1R), which is also known as the Hypocretin I receptor. This protein receptor is localized in the hypothalamus region of the brain and its activation promotes wakefulness in the body.12 Naturally, if MRAP2 can control and activate OX1R, then MRAP2 can be used in drugs that need to activate OX1R.

Interestingly, a newly described Melanocortin type 2 receptor (MC2R) antagonist called GPS1574 was shown to be effective in vitro in an earlier study. Results indicated that it is not a useful MC2R antagonist in vivo. The receptor showed conflicting behavior by being an agonist or an enabler in the presence of high ACTH concentrations but also an antagonist or an opposer in low ACTH hormone concentrations. ${ }^{13}$

$\mathrm{ACTH}$ is also seen as a potent agonist of human MC4R when coexpressed with MRAP2. As both proteins are expressed in the hypothalamic neurons, it provides a target for peripheral ACTH in IS treatments. ${ }^{14}$

Other research on MRAP and MRAP2 seems to offer further information on how stress affects the energy balance and metabolism and how that can in turn lead to obesity and other disorders.

Betamethasone is a cortisol drug that is used to increase susceptibility to spasms. ${ }^{5}$ If it can be shown that prenatal betamethasone priming increases MRAP2 levels, it may be possible to boost the expression of MRAP2 in the hypothalamus so that ACTH can bind more efficiently, helping in the effective treatment in the 45 percent of IS patients. 


\section{Hypothesis}

We hypothesize that MRAP2 expression will be significantly enhanced in the Arcuate Nucleus (ARC) and the Paraventricular Nucleus (PVN) regions of the hypothalamus in prenatally betamethasone primed rats compared to the saline controls. The evidence for the expression can be ascertained by measuring the cell density and the cell area density in the ARC and PVN regions.

Specifically, the null hypothesis, or $\mathrm{H}_{0}$, will be that the cell density and the cell area density will not be statistically significant between the prenatally betamethasone primed and saline control animal groups. The Research Hypothesis, or $\mathrm{H}_{1}$, will be that the cell density and cell area density will be statistically different between the 2 groups.

Figure 3 shows a schematic of the research objective.

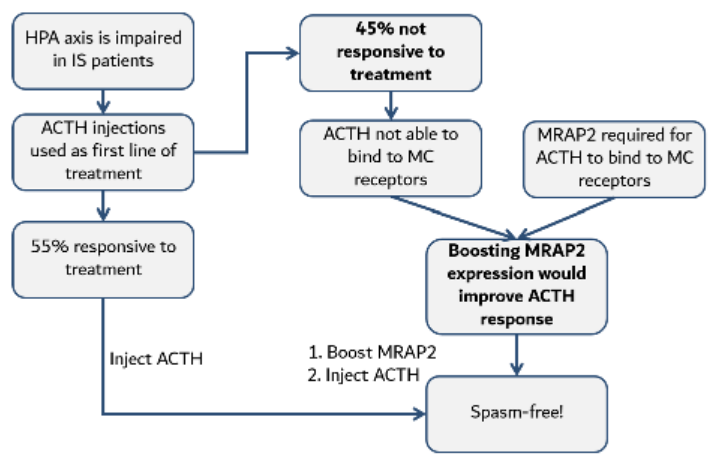

Figure 3. MRAP2 Expression Hypothesis.

\section{Results and Discussion}

A group of 2 male and 2 female rats were exposed to 0.4 $\mathrm{mg} / \mathrm{kg}$ betamethasone on prenatal day 15 , with an identical group exposed to saline as controls. On postnatal day 15, the rats were perfused under deep anesthesia, and the brains were sagittal sectioned. Those steps were performed by qualified scientists in the lab.

Immunohistochemistry (IHC) was performed over 2 days on the sections. Then, they were stained, and the results were analyzed. Pictured here are the blocking buffer, primary antibody, secondary antibody, and Avidin-Biotin solution.

On the first day, after washing and adding a blocking buffer, 300 microliters of the primary rabbit polyclonal antibody were applied to each well for a concentration of 1:1000. The plates were then refrigerated for over 48 hours.

On the second day, after washing the sections, 300 microliters of the anti-rabbit secondary antibody were applied to each well, followed by 300 microliters of Avidin-Biotin solution. Then, 3,3'-diaminobenzidine (DAB) staining was performed to ensure cell visibility during scanning.

8 tissue sections were collected each for ARC and PVN regions. After scanning, the Fiji image analysis software was used to get the cell and cell area density for each image. Finally, the results were tabulated and analyzed.
To determine whether the MRAP2 expression is significant in prenatally betamethasone primed rats, a two-tailed Welch t-test for two samples assuming unequal variances for both the cell density and the cell area density in the ARC and PVN regions was performed. The calculations were done assuming the null hypothesis and that the null hypothesis could be rejected if the t-statistic is greater than the expected value for the confidence level desired. The cell density is calculated as number of cells per unit area of the ARC and PVN region and the cell area density is calculated as ratio of the area of the total cells to the total region area. The MRAP2 expression was detected by the help of the antibody specific to MRAP2 as shown in the methods section.

Figure 4 and Figure 5 show the MRAP2 expression in the ARC area for a prenatally betamethasone primed rat on left and the saline control group on the right for comparison.

The cell area density in the ARC region was statistically significant in the betamethasone group using the 2-tailed $\mathrm{t}$-test with $99 \%$ confidence level $(\mathrm{t}=3.952, \mathrm{P}=0.01)$. The cell number density in the ARC region was statistically significant using the 2-tailed test only at the $94 \%$ confidence level $(\mathrm{t}=$ 2.277, $\mathrm{P}=0.06$ ). The results are indicated in Figure 6 and Figure 7. The Null hypothesis was rejected for the cell area density.

Figure 8 and Figure 9 show the MRAP2 expression in the PVN area for a prenatally betamethasone primed rat on left and the saline control group on the right for comparison.

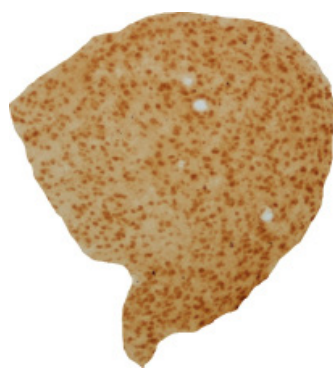

Figure 4. ARC region of betamethasone group rat

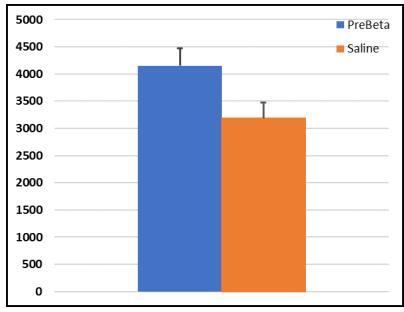

Figure 6. Cell Density in ARC regions (\#cells / mm2). The cell number density in the ARC region was statistically significant using the 2-tailed test only at the $94 \%$ confidence level $(\mathrm{t}=2.277$, $\mathrm{P}=0.06)$.

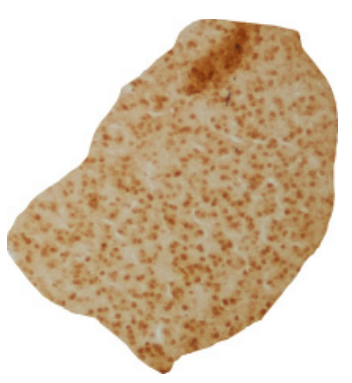

Figure 5. ARC region of saline control group rat

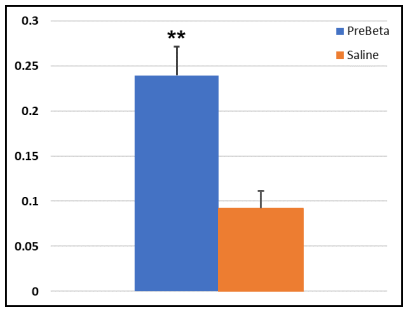

Figure 7. Cell Area Density in ARC region. The cell area density in the ARC region was statistically significant in the betamethasone group using the 2 -tailed $\mathrm{t}$-test with $99 \%$ confidence level $(\mathrm{t}=$ $3.952, \mathrm{P}=0.01)$
On the other hand, both the cell density and the cell area density were not statistically significant in the PVN region using the 2-tailed t-test between the betamethasone and the saline control groups. The t-statistic was 0.48 and the $\mathrm{P}$-val- 
ue was 0.65 for the cell density. The t-statistic was 0.58 and the $\mathrm{P}$-value was 0.58 for the cell area density. The results are indicated in Figure 10 and Figure 11. Therefore, the Null Hypothesis could not be rejected for both the cell density and cell area density in the PVN regions.

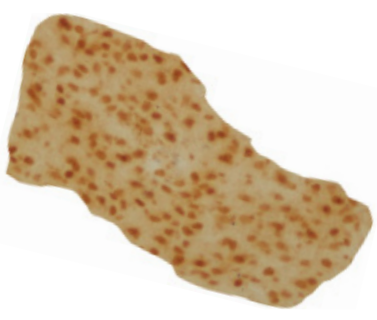

Figure 8. PVN region of betamethasone group rat

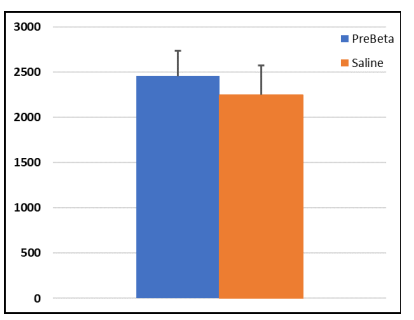

Figure 10. Cell Density in ARC regions (\#cells / mm2). The cell number density in the ARC region was statistically significant using the 2 -tailed test only at the $94 \%$ confidence level $(t=$ 2.277, $\mathrm{P}=0.06$ ).

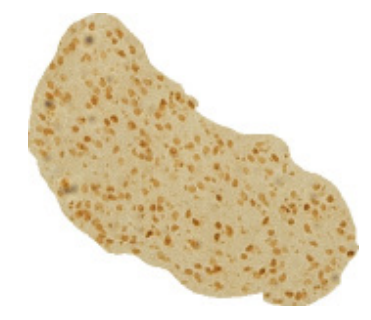

Figure 9. PVN region of saline control group rat

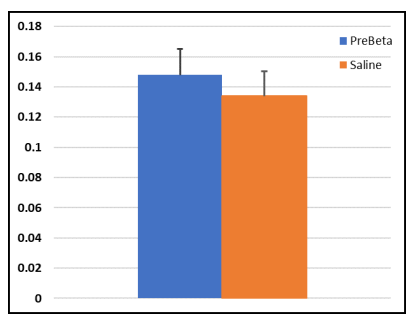

Figure 11. Cell Area Density in ARC region. The cell area density in the $\mathrm{ARC}$ region was statistically significant in the betamethasone group using the 2-tailed $\mathrm{t}$-test with $99 \%$ confidence level $(\mathrm{t}=$ 3.952, $\mathrm{P}=0.01)$.
In prior research, it was shown that MC4R becomes an ACTH receptor after an interaction with MRAP2. Furthermore, as both MC4R and MRAP2 are co-expressed in the hypothalamus, it is suggested that this provides the mechanism for therapeutic actions of ACTH in the treatment of infantile spasms. ${ }^{14}$

In the current experiment, it was discovered that MRAP2 expression is significantly enhanced in the ARC region in prenatally betamethasone primed rats compared to saline controls. The cell area density shows a conclusive significance and the cell density also clearly demonstrated the $6 \%$ significance. One future work would be to reproduce these results with a greater sample size.

Conversely, the current experiment indicates that in the PVN region the MRAP2 expression was not statistically significant compared to the control group. In future experiments, it would be important to not only research as to why there was significance in ARC and not in PVN but also to establish how MRAP2 expression is affected by the onset of seizures using the Velisek IS rat model, which consists of prenatal betamethasone priming and postnatal triggering of spasms with N-methyl-D-aspartic acid (NMDA).

\section{Conclusion}

The cell area density in the ARC region was statistically significant in the prenatally betamethasone primed rats group using the 2 -tailed t-test with a $99 \%$ confidence level. The null hypothesis was rejected for the cell area density.

The cell number density in the ARC region was statistically significant using the 2-tailed test only at the $94 \%$ confidence level; therefore, the null hypothesis could not be rejected.

On the other hand, both the cell density and the cell area density were not statistically significant in the PVN region using the 2-tailed t-test between the prenatally betamethasone primed and the saline control groups. Our research shows that prenatal betamethasone priming enhances the expression of the melanocortin receptor accessory protein 2 (MRAP2) in the ARC region that confirms the sensitivity to ACTH after priming. If it is possible to boost either the activity of MRAP2 proteins by specific agonists or their expression by molecular biology tools to improve the activity of ACTH on their MC receptors, it may be possible to make the $45 \%$ of children that are currently not responsive to ACTH therapy become responsive and help such children be spasm-free.

\section{Methods}

\section{General Information}

A group consisting of 4 male rats and 4 female rats was used in the study. The betamethasone group consisted of 2 male and 2 female rats and the saline control group consisted of the other 2 male and 2 female rats respectively. Both the ARC and $\mathrm{PVN}$ regions were measured in all of the animals and the results were tabulated per section.

The following abbreviations are used throughout this section to identify aspects of the procedure: Immunohistochemistry (IHC), Phosphate-Buffered Saline (PBS), Bovine Serum Albumin (BSA), Normal Goat Serum (NGS), 3,3'-Diaminobenzidine (DAB)

The first group of rats was injected with $0.4 \mathrm{mg} / \mathrm{kg}$ intraperitoneal (IP) betamethasone on prenatal day 15 to serve as the experimental group. The other group of rats was exposed to saline instead of betamethasone as a control group.

\section{Perfusing and Cutting}

To examine the effects of prenatal stress on the MRAP2 gene, rat brains were perfused on postnatal day 15 under deep anesthesia.

The resulting brains were cryoprotected with ascending sucrose concentration (10, 20 and 30\% each for 24 hours) and were then flash-frozen and sagittal sectioned in a cryostat with a thickness of $40 \mu \mathrm{m}$ to access the areas of interest: the paraventricular (PVN) \& arcuate (ARC) nuclei of the hypothalamus. The slices remained immersed in PBS while immunohistochemistry was performed.

\section{Immunohistochemistry Day 1}

IHC is a multi-step method used to ensure antibody visibility as well as the ability to view neurons from under the microscope.

The first step following the brain sectioning was the concentration testing of the primary antibody to ensure optimal 
contrast for IHC. IHC was performed with antibody ratios $1: 250,1: 500,1: 1000$, and a negative control, using brains treated with Kainic acid, which are known to express MRAP2. Kainic acid brains were also used as a positive control during IHC to verify that the process was working correctly (Figure 12 and Figure 13). After considering the concentrations of $1: 250,1: 500$, and 1:1000, the microscope images determined that 1:1000 offered the best contrast between the background and the stained cells in the foreground (Figure 14). It was decided to move forward with a concentration of 1:1000 of the primary antibody to PBS. This concentration was used when working with the experimental tissue. $\mathrm{DAB}$ was used to stain the tissue and a set of reference slides were stained with cresyl violet (which stains all nuclear bodies) to aid with microscopy in addition to Paxinos' Rat Brain Atlas, 6th ed.

Avidin-Biotin IHC was used. The primary antibody was the Proteintech MRAP2 Rabbit Polyclonal antibody (catalog number 17259-1-AP) and my secondary antibody was anti-rabbit, raised in goats.

Avidin-Biotin IHC exploits the high affinity between biotin and avidin. Biotin can be linked to the secondary (anti-rabbit) antibody, i.e., the biotinylated antibody, localizing the site of the antigen by tagging the primary antibody. Then, avidin conjugated to horseradish peroxidase was added. It bound to the biotinylated antibody and localized the peroxide molecule at the site of the antigen. Finally, the antigen can be detected by using a substrate for peroxidase.

For the first day of IHC, the sagittal slices were washed three times on ice while changing the $0.01 \mathrm{M}$ PBS every 10 minutes and allowing the slices to wash on the shaker. Then, the blocking buffer was prepared and placed, which is composed of $20 \%$ BSA (1\% of final solution), NGS (10\% of the final solution), and Triton X-100 ( $0.3 \%$ of final solution). 300 microliters of the resulting blocking buffer were then applied to each well and the plates were placed on the shaker for 60 minutes. Then, the primary antibody was applied to each well at an amount of 300 microliters per well (concentration 1:1000). The one designated negative control per plate of approximately 19 sagittal slices was kept in the previous blocking buffer and was not in-

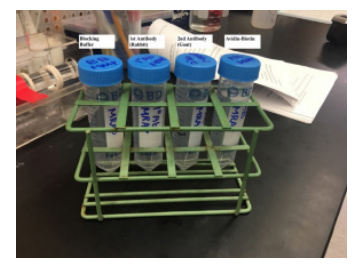

Figure 12. Concentration Testing Setup

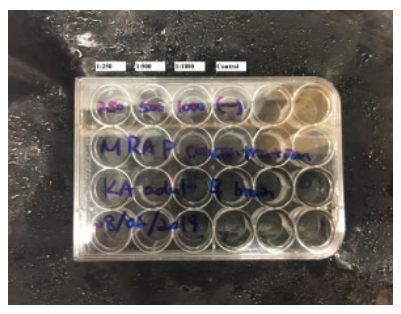

Figure 13. Concentration Testing Samples the primary antibody. The resulting wells were then refrigerated for over 48 hours before beginning IHC day 2.

\section{Immunohistochemistry Day 2}

On the second day of IHC, the plates were removed from the fridge and were washed again in PBS three times, while on the shaker for 10 minutes each. Using a pipette, the PBS from each well was carefully removed to keep the tissue intact.

Next, the secondary antibody was applied. The secondary antibody was anti-rabbit, and it made up $0.5 \%$ of the final secondary solution. The solution also contained $4 \%$ of $20 \%$ BSA and $95.5 \%$ PBS. The plates were then placed on the shaker for 60 minutes and then washed.

Avidin-Biotin solution consists of $1 \%$ Avidin and $1 \%$ of Biotin, with the remaining $98 \%$ of the solution consisting of PBS. 300 microliters of the solution were placed into each well after successfully removing the cleansing PBS, and the plates were then placed on the shaker again for another 60 minutes.

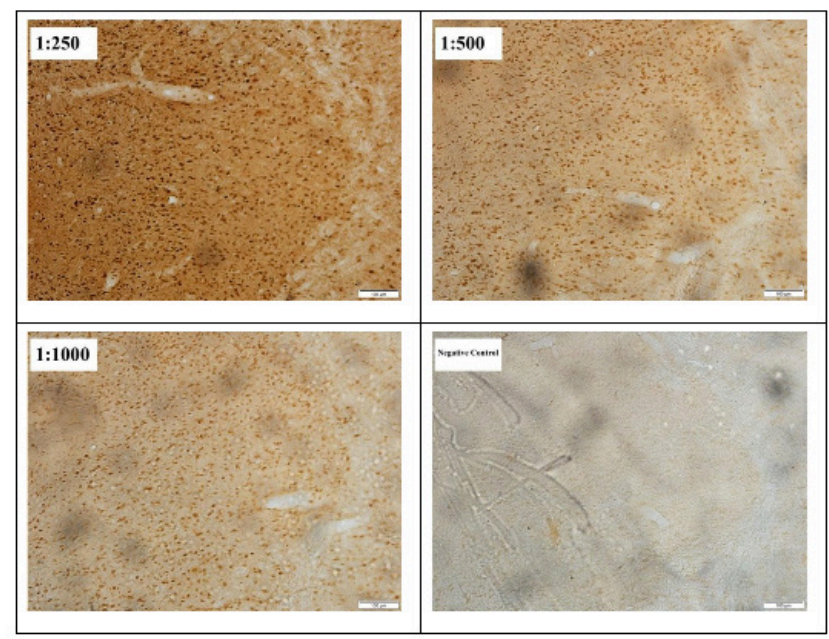

Figure 14. Scans at various concentrations of antibody. Dilution 1:1000 offers the best contrast between the background and the stained cells in the foreground.

\section{DAB Staining}

The $\mathrm{DAB}$ portion of the IHC requires personal protection equipment as well as a chemical fume hood. The creation of the $\mathrm{DAB}$ solution, as well as the staining process, took place fully underneath the fume hood. $25 \%$ of Reagent A, which was the oxidized form of benzene, and $25 \%$ of Reagent $\mathrm{B}$, which was hydrogen peroxide, were combined with a remainder of $50 \%$ PBS. As the solution remained in ice, the PBS was quickly extracted from three wells at a time and applied 300 microliters of DAB solution to each well. During the concentration testing, it was noted that three minutes was preferable in achieving the correct amount of darkness in the staining. A timer was set to three minutes and the plate was gently moved throughout that period to ensure even staining. After three minutes, PBS was instantly applied to each of the three wells to dilute the solution. Then, the diluted solution was removed using a pipette and the stained tissue was then immersed in new PBS. This procedure was repeated until each plate was stained. The waste solution was properly disposed of in the hazardous waste 
bucket within the lab and the gloves were also properly disposed of.

\section{Mounting and Imaging}

The resulting plates were left in PBS for a few days before mounting. Five sagittal sections were placed on each gelatinized slide, and the slides were cover-slipped using xylene and glue under the fume hood.

The resulting slides for the regions of interest were imaged at 40x and 100x magnification using a Nikon Eclipse microscope and an Olympus camera with the cellSens Dimension software. Structures that were too large for the field of view were montaged using the MIA feature of the microscopy software.

The ARC and PVN regions of the hypothalamus were scanned to detect differences between the prenatally betamethasone primed and saline tissues.

\section{Image Analysis}

The slide images were analyzed using the Fiji software package. First, the regions were marked on each image. Next, the $\mathrm{ROI}$ tool was used to select and measure the regions. Once the region was measured, the cells and cell density were measured using the 'analyze region' option. Where necessary, appropriate band-pass filtering was done to ensure proper measurements. The measurements were used to derive the number of stained cells as well as the area of the cells per unit area (density of staining) in each tissue sample.

\section{- Acknowledgements}

Research was conducted at New York Medical College (NYMC) in Valhalla, NY.

The author would like to thank Dr. Libor Velisek for providing the opportunity and helping with the research in his lab at NYMC.

\section{References}

1. NIH. Infantile Spasms Information Page | National Institute of Neurological Disorders and Stroke https://www.ninds.nih.gov/Disorders/ All-Disorders/Infantile-Spasms-Information-Page.

2. NORD. Infantile Spasms - NORD (National Organization for Rare Disorders) https://rarediseases.org/physician-guide/infantilespasms/(accessed May 27, 2019)

3. Camfield, P.; Camfield, C. Long-Term Prognosis for Symptomatic (Secondarily) Generalized Epilepsies: A Population-Based Study. Epilepsia 2007, 48 (6), 1128-1132. https://doi.org/10.1111/ j.1528-1167.2007.01072.x.

4. Baram, T. Z.; Hirsch, E.; Snead, O. C.; Schultz, L. CorticotropinReleasing Hormone-Induced Seizures in Infant Rats Originate in the Amygdala. Annals of Neurology 1992, 31 (5), 488-494. https://doi.org/10.1002/ana.410310505.

5. Velíšek, L.; Jehle, K.; Asche, S.; Velíšková, J. Model of Infantile Spasms Induced by N-Methyl-D-Aspartic Acid in Prenatally Impaired Brain. Annals of Neurology 2007, 61 (2), 109-119. https://doi.org/10.1002/ana.21082.

6. Rouault, A. A. J.; Srinivasan, D. K.; Yin, T. C.; Lee, A. A.; Sebag, J. A. Melanocortin Receptor Accessory Proteins (MRAPs): Functions in the Melanocortin System and Beyond. Biochimica et Biophysica Acta (BBA) - Molecular Basis of Disease 2017, 1863 (10), 2462-2467. https://doi.org/10.1016/j.bbadis.2017.05.008.

7. Sebag, J. A.; Hinkle, P. M. Melanocortin-2 Receptor Accessory Protein
MRAP Forms Antiparallel Homodimers. Proceedings of the National Academy of Sciences 2007, 104 (51), 20244-20249. https://doi.org/10.1073/pnas.0708916105.

8. Liu, T.; Elmquist, J. K.; Williams, K. W. Mrap2: An Accessory Protein Linked to Obesity. Cell Metabolism 2013, 18 (3), 309-311. https://doi.org/10.1016/j.cmet.2013.08.016.

9. Asai, M.; Ramachandrappa, S.; Joachim, M.; Shen, Y.; Zhang, R.; Nuthalapati, N.; Ramanathan, V.; Strochlic, D. E.; Ferket, P.; Linhart, K.; et al. Loss of Function of the Melanocortin 2 Receptor Accessory Protein 2 Is Associated with Mammalian Obesity. Science 2013, 341 (6143), 275-278. https://doi.org/10.1126/science.1233000.

10.Clark, A. J. L.; Chan, L. F. Promiscuity among the MRAPs. Journal of Molecular Endocrinology 2017, 58 (3), F1-F4. https://doi.org/10.1530/jme-17-0002.

11.Margioris, A. N.; Christos Tsatsanis. ACTH Action on the Adrenals https://www.ncbi.nlm.nih.gov/books/NBK279118/ (accessed Aug 5, 2019).

12.Rouault, A. A. J.; Lee, A. A.; Sebag, J. A. Regions of MRAP2 Required for the Inhibition of Orexin and Prokineticin Receptor Signaling. Biochimica et Biophysica Acta (BBA) - Molecular Cell Research 2017, 1864 (12), 2322-2329. https://doi.org/10.1016/j.bbamcr.2017.09.008.

13.Goldenberg, A. J.; Gehrand, A. L.; Waples, E.; Jablonski, M.; Hoeynck, B.; Raff, H. Effect of a Melanocortin Type 2 Receptor (MC2R) Antagonist on the Corticosterone Response to Hypoxia and ACTH Stimulation in the Neonatal Rat. American Journal of Physiology-Regulatory, Integrative and Comparative Physiology 2018, 315 (1), R128-R133. https://doi.org/10.1152/ajpregu.00009.2018.

14.Soletto, L.; Hernández-Balfagó, S.; Rocha, A.; Scheerer, P.; Kleinau, G.; Cerdá-Reverter, J. M. Melanocortin Receptor Accessory Protein 2-Induced Adrenocorticotropic Hormone Response of Human Melanocortin 4 Receptor. Journal of the Endocrine Society 2018, 3 (2), 314-323. https://doi.org/10.1210/js.2018-00370.

\section{Authors}

Nitin Seshadri is currently a senior at Somers High School. His biggest interests are science (especially the workings of the brain), writing, and playing violin. He is planning to major in the pre-med track and ultimately go to medical school. 